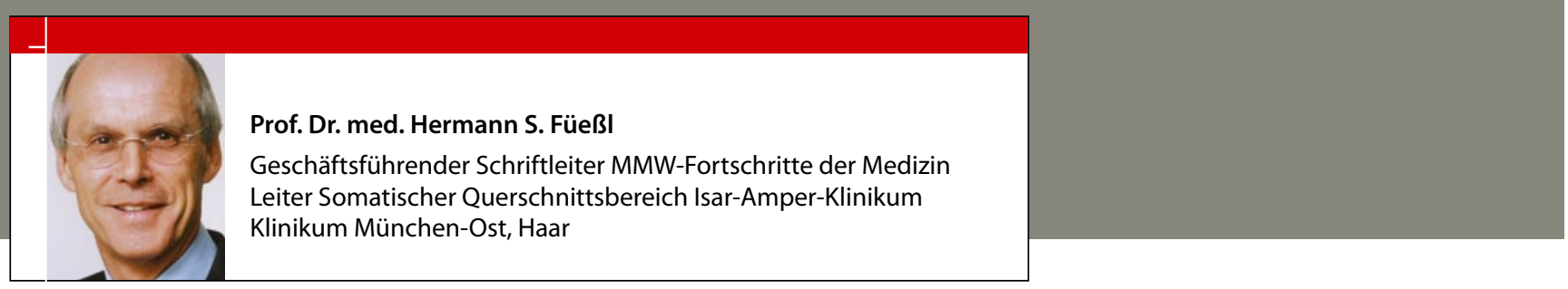

\title{
6. Viel mehr als PAVK
}

- Sine vasa, nulla vita! In dichterischer Freiheit und grammatikalisch nicht ganz korrekt darf man mit Fug und Recht behaupten, dass ohne Blut kein Leben sei, und Blut kommt eben über die Gefäße. Dieses Axiom bedeutet für die Wissenschaft von den Gefäßen, die Angiologie, umfassenden Anspruch und Tragik zugleich. Im Grunde sind nämlich bei allen Krankheiten Blutgefäße involviert, und somit könnte man Schlaganfall und Herzinfarkt, Bronchial- und Kolonkarzinom, Diabetes und COPD, rheumatoide Arthritis und Lupus erythematodes auch als Gefäßkrankheiten bezeichnen. Allerdings orientiert sich die Organisation unserer Medizin im Wesentlichen an den betroffenen Organen, was bedeutet, dass das Herz den Kardiologen, das Gehirn den Neurologen, die Nieren den Nephrologen und die Gelenke den Rheumatologen "gehören".

Darin liegt für die Angiologie eine gewisse Tragik, da sie sich organisch nur schwer verorten lässt. Lediglich der Gefäßabschnitt zwischen Leistenband und Sprunggelenk bzw. zwischen Schulter und Hand wird ihr als eigenes Terrain in Form der peripheren arteriellen Verschlusskrankheit (PAVK) zugestanden. Und selbst hier toben die Verteilungskämpfe, vor allem mit der Gefäßchirurgie und der Radiologie. Nachdem die PAVK eine häufige Komplikation des Diabetes mellitus ist, wurde die Angiologie - nicht immer zu ihrem Vorteil - an vielen Krankenhäusern den Abteilungen für Diabetologie und Stoffwechsel zugeschlagen.

Gefäßerkrankungen können sich an verschiedensten Organen manifestieren und dazu führen, dass unterschiedliche medizinische Disziplinen damit beschäftigt werden, ohne dass häufig eine gefäßspezifische Ausbildung und der um- fassende Überblick vorhanden sind. Immerhin wurde in Würdigung der epidemiologischen Bedeutung und der Komplexität der Materie 1992 die Angiologie als jüngstes Teilgebiet der Inneren Medizin mit einer eigenen Facharztbezeichnung etabliert. Damit fanden nach über 40 Jahren die Bemühungen des Vaters der deutschen Angiologie, Max Ratschow, eine späte Anerkennung.

Dennoch sind die Weiterbildungsmöglichkeiten sehr begrenzt, und entsprechend mangelt es an Nachwuchs. Querschnittsfächer haben es eben nicht leicht. Methodisch gibt es traditionell Querelen mit der interventionellen Radiologie, topografisch mit der Neurologie bei den hirnversorgenden Gefäßen und mit der Dermatologie bei den Venenerkrankungen, therapeutisch mit der Gefäßchirurgie. Obwohl im Sinne des Patienten Kooperation angebracht wäre, betreibt man, je nach Sichtweise, wechselseitige Wilderei.

Doch es gibt auch vielversprechende Kooperationsmodelle in Form sog. gefäßmedizinischer Zentren, in denen ein Kernteam (Angiologen, Gefäßchirurgen und Radiologen, ergänzt durch Kardiologen, Neurologen, Diabetologen, Podologen bis zum orthopädischen Schuhmachermeister) seine Expertise fachübergreifend und interaktiv bei der Behandlung von Gefäßpatienten einbringt. Welche komplexen Krankheitsbilder den Angiologen beschäftigen, zeigt der folgende Beitrag „Kongenitale vaskuläre Anomalien“ sowie der Beitrag "Nicht immer nur PAVK. Arterielle Verschlusserkrankungen ohne Atherosklerose", der in einer der nächsten Ausgaben publiziert wird. Angiologie ist eben viel mehr als Claudicatio intermittens und schwarze Füße.

12-jähriges Mädchen mit vermehrtem Längenwachstum des rechten Arms auf dem Boden einer arterio-venösen Malformation, die von der Schulter bis zu den Fingern reicht (Sterne). 